\title{
Aspectos das manifestações da síndrome pós-COVID-19: uma revisão narrativa
}

\author{
Aspects of post-COVID-19 syndrome manifestations: a narrative review
}

Aspectos de las manifestaciones posteriores al síndrome COVID-19: una revisión narrativa

Mércia Alexandra Amorim Silveira ${ }^{1 *}$, Bruno Aguiar Martins ${ }^{1}$, Larissa Silva Feres Gomes Chamon ${ }^{1}$, Ana Elisa Dias Diniz ${ }^{1}$, Jênifer Bicalho de Assis ${ }^{1}$, Luísa Dias Toledo Ferreira ${ }^{1}$, Mariana Fernandes de Sousa Macêdo ${ }^{1}$, Maressa Precioso Verdin ${ }^{1}$, Waléria Pimenta Cunha Pereira ${ }^{1}$, Henrique de Castro Mendes ${ }^{2}$.

\section{RESUMO}

Objetivo: Realizar uma revisão bibliográfica sobre a Síndrome Pós-COVID-19 (SPC). Revisão bibliográfica: A SPC é um termo utilizado na literatura para designar sinais e sintomas físicos e psicológicos debilitantes e persistentes, além do tempo usual, apresentados por pacientes que já se encontram na fase de recuperação da infecção pelo SARS-CoV-2. Alguns fatores de risco dessa síndrome são: idade avançada, presença de comorbidades, sexo feminino e internação hospitalar. Entre as manifestações clínicas do COVID-19 longo, que é o outro nome utilizado para a SPC, podem-se citar: fadiga, dispneia, dor torácica e sintomas neuropsiquiátricos. Ademais, também são relatadas complicações decorrentes da SPC, como fibrose pulmonar, bronquiectasia e fenômenos tromboembólicos. Acerca do prognóstico desta síndrome, os dados publicados apontam para uma boa evolução geral dos pacientes, com a resolução das complicações. Considerações finais: A SPC é uma síndrome multissistêmica que pode afetar pacientes recuperados da COVID-19 e impactar negativamente em sua qualidade de vida. Portanto, é importante conhecer a doença, suas manifestações, o diagnóstico, tratamentos e prognóstico.

Palavras-chave: Infecção por SARS-CoV-2, COVID-19, Pandemia por COVID-19.

\begin{abstract}
Objective: To perform a literature review on Post COVID-19 Syndrome (PCS). Bibliographic review: PCS is a term used in the literature to designate debilitating and persistent physical and psychological signs and symptoms, beyond the usual time, presented by patients who are already in the recovery phase of SARSCoV-2 infection. Some risk factors for this syndrome are: advanced age, presence of comorbidities, female gender and hospitalization. Among the clinical manifestations of long COVID-19, we can mention: fatigue, dyspnea, chest pain and neuropsychiatric symptoms. Furthermore, complications resulting from PCS, such as pulmonary fibrosis, bronchiectasis and pulmonary embolism, are also reported. About the prognosis of this syndrome, published data point to a good overall evolution of patients, with symptomatic relief. Final considerations: The PCS is a multisystemic syndrome that can arise in patients recovering from COVID-19 and negatively impact their quality of life. Therefore, it is important to know the disease, its signs, diagnosis, treatments and prognosis.
\end{abstract}

Keywords: SARS-CoV-2, COVID-19, COVID-19 pandemic.

\section{RESUMEN}

Objetivo: Realizar una revisión de la literatura sobre el Síndrome Post-COVID-19 (PCS). Revisión bibliográfica: PCS es un término utilizado en la literatura para designar signos y síntomas físicos y

\footnotetext{
${ }^{1}$ Faculdade de Medicina do Vale do Aço (UNIVAÇO), Ipatinga - MG. *E-mail: merciasilveira7@gmail.com

2 Universidade Federal de Minas Gerais (UFMG), Belo Horizonte - MG
}

SUBMETIDO EM: 11/2021 
psicológicos debilitantes y persistentes, más allá del tiempo habitual, presentados por pacientes que ya se encuentran en la fase de recuperación de la infección por SARS-CoV-2. Algunos factores de riesgo de este síndrome son: edad avanzada, presencia de comorbilidades, sexo femenino y hospitalización. Entre las manifestaciones clínicas del COVID-19 prolongado, que es el otro nombre que se utiliza para CPS, podemos mencionar: fatiga, disnea, dolor torácico y síntomas neuropsiquiátricos. Además, también se describen las complicaciones derivadas de la CPS, como la fibrosis pulmonar, las apuntan a una buena evolución general de los pacientes, con resolución de complicaciones. Consideraciones finales: EI CPS es un síndrome multisistémico que puede afectar a los pacientes recuperados del COVID-19 e impactar negativamente en su calidad de vida. Por tanto, es importante conocer la enfermedad, sus manifestaciones, diagnóstico, tratamientos y pronóstico.

Palabras clave: Infección por SARS-CoV-2, COVID-19, Pandemia de COVID-19.

\section{INTRODUÇÃO}

A pandemia ocasionada pelo Coronavírus 2 da Síndrome Respiratória Aguda Grave (SARS-CoV-2) afetou de maneira devastadora todas as camadas da sociedade. Iniciada no mês de dezembro de 2019, em Wuhan, na China, a pandemia da doença do coronavírus 2019 (COVID-19) trouxe diversos desafios no contexto sanitário, devido à sua gravidade, rapidez de transmissão e apresentação clínica ampla. Sabe-se que até 30 de outubro de 2021, havia mais de 245 milhões de casos confirmados em todo o mundo, sendo registrados cerca de 4,97 milhões de óbitos (ORGANIZAÇÃO MUNDIAL DA SAÚDE, 2021).

Em estudos recentes, os sintomas mais comuns apresentados são dispneia, cefaleia, tosse, queda de cabelo, ansiedade, anosmia, ageusia, dificuldade para dormir, dor no peito, sintomas gastrointestinais e palpitações (KOROMPOKI E, et al., 2021; ORONSKY B, et al., 2021). Inicialmente, vários esforços foram destinados ao controle da infecção, a fim de evitar o esgotamento das trocas gasosas e da capacidade pulmonar, objetivando um menor comprometimento das funções vitais. Contudo, com o passar do tempo, novos estudos verificaram que o SARS-CoV-2 é mais do que somente uma síndrome respiratória, já que a resposta inflamatória ao vírus gera a produção de altos níveis de substâncias químicas endógenas, o que leva a alterações teciduais, imunológicas e hematológicas (ANDRADE BS, et al., 2021).

Diante de todos esses fatores mencionados, essa doença pode ser caracterizada como multissistêmica a longo prazo, pois afeta consideravelmente diversos órgãos, como pulmão, coração e cérebro, e, por conta disso, gera um processo de reabilitação mais complexo e prolongado (MARTíNEZ WC, et al., 2021). Além disso, sabe-se que, dentre os órgãos acometidos, aquele que apresenta a maior taxa de complicações é o pulmão, e, dessa maneira, dependendo do quadro clínico e evolução do paciente, desordens como: fibrose pulmonar, função pulmonar anormal, incluindo capacidade de difusão de monóxido de carbono reduzida, exames de imagem com achados de consolidação e fibrose pulmonares e sequelas de embolia podem ser encontradas (KOROMPOKI E, et al., 2021; MONTANI D, et al., 2021).

Outro aspecto importante está relacionado aos fatores de risco presentes no indivíduo, como, por exemplo, condições desfavoráveis pré-existentes, uma vez que estão relacionadas a um prejuízo na recuperação clínica e bom prognóstico do paciente. Desse modo, vale ressaltar que a ocorrência de aumento transitório da resistência à insulina em pacientes com diabetes e hiperglicemia é um fator de risco independente para maior gravidade da infecção pelo vírus (KHUNTI K, et al., 2021). Outros fatores também foram associados a um pior desfecho, sendo eles discutidos posteriormente.

Em relação às sequelas, as manifestações podem ser de curto, médio ou longo prazo e, sendo assim, o seu manejo deve ser realizado de forma individual (BECKER RC, 2021). Na visão de Vehar S, et al. (2021), o tratamento dos pacientes deve ser realizado de forma multidisciplinar, por meio da observação e avaliação clínica, bem como pela realização de um acompanhamento rigoroso dos pacientes que foram internados. Para isso, a realização da triagem virtual tem se mostrado uma ferramenta de grande valia, uma vez que, caso seja observada a persistência dos achados clínicos prévios, o encaminhamento para a realização de novos exames e a condução ao especialista se faz necessária. 
O presente artigo teve como objetivo expor dados relevantes sobre a Síndrome Pós-COVID-19 (SPC) vivenciada em tempos atuais, visto que tal sequela tem trazido preocupações crescentes, devido ao grande número de casos relatados e, também, pela necessidade de compreender os mecanismos envolvidos na doença e os novos manejos clínicos.

\section{REVISÃO BIBLIOGRÁFICA}

\section{Conceito}

A síndrome pós-COVID-19, também chamada de "COVID-19 longo", é um termo utilizado pelos pesquisadores para designar uma série de sinais e sintomas físicos e psicológicos debilitantes e persistentes, além do tempo usual, apresentados por pacientes que já se encontram na fase de recuperação da infecção pelo SARS-CoV-2 (MARTIMBIANCO ALC, et al., 2021).

Além disso, aparenta ser uma doença multissistêmica que ocorre após um acometimento agudo, com risco estimado em $10-35 \%$ dos pacientes de modo geral e $85 \%$ para aqueles que foram hospitalizados (PAVLI A, et al., 2021). Infelizmente, segundo Becker RC (2021), muitos desses que necessitaram de hospitalização serão readmitidos até dois meses após alta hospitalar, apesar de a frequência e a gravidade dos sintomas a longo prazo, segundo Rello J, et al. (2021), não estarem diretamente ligados ou exclusivos aos pacientes graves.

É importante destacar que essa condição clínica recebe critérios temporais que variam de 3 a 24 semanas após a fase aguda, sendo definida como pós-aguda quando se estende além de 3 semanas e crônica quando perdura por mais de 12 semanas (MARTIMBIANCO ALC, et al., 2021). Essa definição de tempo possibilita um melhor delineamento das características clínicas apresentadas pelos pacientes, o que gera um aperfeiçoamento do cuidado primário e secundário, além de um direcionamento adequado dos recursos, de modo a refinar a assistência à saúde (IQBAL FM, et al., 2021).

\section{Fatores de risco}

Assim como diversas outras condições clínicas, constatou-se que a síndrome pós-COVID-19 possui alguns fatores de risco que propiciam o seu desenvolvimento. De acordo com Martimbianco ALC, et al. (2021), características como idade avançada, a presença de comorbidades, quadro clínico mais grave, sexo feminino, internação hospitalar e a necessidade de oxigênio suplementar são fatores que foram relacionados ao "COVID-19 longo". Além disso, o isolamento social, o sedentarismo e a insegurança social e econômica também contribuem para as suas manifestações clínicas e psicológicas.

Um estudo publicado por Iqbal FM, et al. (2021) verificou que os pacientes que tinham uma carga de sintomas mais elevada durante a fase inicial da infecção tinham mais chances de fadiga persistente. Em contrapartida, Sykes DL, et al. (2021) sugere que há pouca relação entre a gravidade da doença aguda e a probabilidade de desenvolver manifestações a longo prazo.

Além das condições já descritas, John AE, et al. (2021), propõe que história prévia de patologias respiratórias crônicas, doenças hepáticas, neoplasias hematológicas e desordens neurológicas corroboram para um aumento do risco de complicações e mortalidade por COVID-19. Por fim, existem outros fatores que também estão associados a resultados insatisfatórios em pessoas acometidas pelo "COVID-19 longo", como obesidade, hiperglicemia, doenças cardiovasculares e renais. Sendo assim, o gerenciamento clínico adequado é fundamental para garantir a melhoria das funções metabólicas e uma boa recuperação desses pacientes (KHUNTI K, et al., 2021).

\section{Fisiopatologia}

O mecanismo fisiopatológico da síndrome pós-COVID-19 e suas consequências a longo prazo é ainda uma incógnita, principalmente por se tratar de relatos com cerca dezenas de manifestações sintomatológicas diferentes (POENARU S, et al., 2021). Por este motivo, existe uma dificuldade para determinar um mecanismo lógico de como a doença avança, já que o acometimento é multissistêmico, o que tende a nos levar para uma longa caminhada em busca de quando e como cada sistema é lesado (PAVLI A, et al., 2021). 
Tomando como base os relatos de outras infecções virais, como a do vírus Epstein-Barr (EBV), que apresenta similaridades ao "COVID-19 longo", foram encontrados dados que possibilitam a inferência de que: a resposta, durante a infecção do coronavírus, poderia seguir duas ramificações de atividade, o que seria a inflamação crônica e o fenômeno autoimune; sendo a última devido à "tempestade de citocinas" ou hipercitocinemia, que é a resposta exacerbada do das defesa orgânicas, atenuando a resposta Th1/T17 com alterações de funcionalidade dos leucócitos natural killer, em concomitância dos linfócitos $B$ auto-reativos (FAJGENBAUM D e JUNE C, 2020).

Ainda sobre os estudos de Poenaru S, et al. (2021), na medida em que há progressão da hipercitocinemia, haveria a desmielinização com consequentes lesões neuronais que levariam a uma alteração da condução nervosa e resposta de fluxo sanguíneo. Somado a isso, ações sobre a atividade mitocondrial explicaria, em partes, todo este mecanismo, com a alteração do metabolismo aeróbico, uma tendência para uma anaerobiose/acidose metabólica em concomitância da diminuição do estoque de coenzima $Q$ e aumento das espécies reativas de oxigênio.

Raciocinando em cima dos estudos de Poenaru S, et al. (2021) e Pavli A, et al. (2021), foi possível encontrar lógica de seus trabalhos, ao compararmos com as proposições de Martimbianco ALC, et al. (2021), onde a idade avançada e demais fatores fisiológicos podem estar relacionados com a síndrome pós-COVID19, já que a natureza dessas variáveis está relacionada com quadros de autoimunidade, dores crônicas e demais alterações notadas, também, no estudo de Khunti K, et al. (2021).

\section{Manifestações clínicas e prognóstico}

A incidência da síndrome pós-COVID-19 em pacientes tratados em regime ambulatorial varia entre $10 \mathrm{e}$ $35 \%$, enquanto em indivíduos hospitalizados chega a quase $80 \%$. Assim, pode-se inferir que esse distúrbio não se limita a casos de COVID-19 aguda grave (que necessitam de internação). Sabendo disso, é interessante ter o conhecimento das manifestações clínicas do "COVID-19 longo" em qualquer paciente acometido pela doença. Dentre elas, destacam-se fadiga, dispneia, dor torácica, manifestações mentais (depressão e ansiedade), comprometimento cognitivo, distúrbios cerebrovasculares e/ou neurológicos centrais (vasculite, acidente vascular cerebral isquêmico e encefalites), distúrbios neurológicos periféricos (disfunção olfativa e gustativa) (PAVLI A, et al., 2021).

Além disso, também são relatadas complicações decorrentes do acometimento pulmonar da COVID-19 como alterações intersticiais fibróticas e não fibróticas, pneumonia em organização, bronquiectasia, embolia pulmonar, e outras como isquemia cardioembólica aguda de membros, artrites, lúpus eritematoso sistêmico, síndrome de Guillain-Barré, miocardite, lesão renal aguda, lesão hepática e doença de Graves, podendo haver outras ainda não relatadas (KOROMPOKI E, et al., 2021).

Além do comprometimento do sistema respiratório na síndrome pós-COVID-19, estudos encontraram uma gama de alterações sistêmicas consequentes à infecção pelo SARS-CoV-2. Nalbandian A, et al. (2021) identificou sinais nos sistemas cardiovascular, neurológico, renal, endócrino, gastrointestinal e dermatológico. Tolba M, et al. (2021) realizou um estudo com 287 pacientes egípcios que se recuperaram de COVID-19 e encontrou que $89,2 \%$ deles apresentaram alguma manifestação sintomática, sendo a fadiga mais comum $(72,8 \%)$, seguida de ansiedade $(38 \%)$, artralgias $(31,4 \%)$ e cefaleias recorrentes $(28,9 \%)$, e que houve melhora com tratamento em $67,2 \%$ dos casos.

Higgins V, et al. (2021) introduz o conceito da síndrome pós-terapia intensiva, que apresenta como manifestações os sintomas neuropsiquiátricos relatados na literatura, em conjunção com artralgia, perda de massa muscular, disfunção diafragmática, lesão laríngea, disfagia e disfonia, que são observados em pacientes graves com recuperação e alta da unidade de terapia intensiva. Há vários fatores de risco associados, como a intensidade e duração da sedação, uso de bloqueadores neuromusculares, imobilidade, necessidade de ventilação mecânica, toxicidade viral direta e a falta de contato com outras pessoas. Outrossim, observou-se semelhança na descrição dos sistemas orgânicos mais afetados pela síndrome pósCOVID-19 com a literatura estabelecida. 
Satterfield BA, et al. (2021), em sua revisão acerca do comprometimento cardiovascular a longo prazo pós-COVID-19, especula que possa haver um aumento de casos de disfunção sistólica e insuficiência cardíaca devido à alta ocorrência de lesão miocárdica pelo SARS-CoV-2 e sua associação a desfechos desfavoráveis a curto prazo. Segundo o estudo, o vírus está associado à síndrome coronariana aguda, miocardite, disfunções ventriculares, arritmias, trombose venosa profunda, tromboembolismo pulmonar e pericardite, decorrentes da resposta imunológica excessivamente agressiva e estado de hipercoagulabilidade.

Zacharias $\mathrm{H}$, et al. (2021) descreve as complicações reumatológicas da COVID-19, dividindo-as em artrites inflamatórias, doenças do tecido conjuntivo e vasculites. Na literatura revista, foram descritos casos de vasculites, sobretudo de grandes vasos, podendo ter papel no processo patológico inicial e nos sintomas persistentes. Dentre as doenças do tecido conjuntivo, foram relatados casos de síndromes lupus-like, miosite viral e rabdomiólise consequente ao processo inflamatório. Em relação às artrites, houve relatos de casos de indivíduos que desenvolveram artrite reumatoide pós-infecção.

Outro exemplo de acometimento pós-COVID-19 é a Lesão Renal Aguda (LRA), muito comum em pacientes que contraíram a forma grave da doença e que necessitam de internação em UTI. Como dito por Fajgenbaum D e June C (2020), uma das causas é a própria reação do sistema imune em combate ao SARS-CoV-2, gerando uma resposta intensa capaz de causar lesão renal. Nestes casos, há necessidade de acompanhamento com a nefrologia (MEIER P, et al., 2011 apud NALBANDIAN A, et al., 2021).

Becker RC (2021) apresenta uma classificação das manifestações do "COVID-19 Longo" em cinco categorias, com seus respectivos subtipos: Tipo 1: diferentes períodos de recuperação e reabilitação que se correlacionam diretamente com a gravidade da infecção, as lesões de órgão-alvo e condições médicas préexistentes. Tipo 2: sintomas que continuam 6 semanas após a infecção inicial. Tipo 3: recuperação quase total após a infecção inicial, mas com retorno dos sintomas por um período maior que três meses (tipo $3 \mathrm{~A}$ ) ou maior que seis meses (tipo 3B). Tipo 4: assintomáticos na realização do teste SARS-CoV-2 positivo, mas desenvolvem sintomas num período de 1-3 meses (tipo 4A) ou após 3 meses (tipo 4B). Tipo 5 (raro): assintomáticos ou minimamente sintomáticos durante o teste de SARS-CoV-2 positivo e sofrem morte súbita nos 12 meses subsequentes.

De forma geral, cruzando os dados dos artigos de Becker RC (2021), Korompoki E, et al. (2021) e Pavli A, et al. (2021), acerca do prognóstico da síndrome pós-COVID-19, as evidências apontam para uma boa evolução na maioria dos casos. No entanto, Malik P, et al. (2022), em sua meta-análise, apontou que a SPC está associada a pior qualidade de vida e persistência sintomática a longo prazo, e destaca a incerteza de conhecimento sobre os fatores de risco devido ao foco no combate à pandemia. Sendo assim, com uma população numerosa de pacientes que se recuperaram da COVID-19 e alguns desenvolveram a SPC, é importante realizar investigações sobre o tema, de modo a desenvolver estratégias de seguimento e tratamento adequadas.

\section{Diagnóstico}

A síndrome pós-COVID-19 apresenta caráter de acometimento orgânico multissistêmico e alguns pesquisadores identificaram-na como a interação de pelo menos 4 entidades clínicas distintas: as síndromes pós-terapia intensiva, fadiga pós-viral e COVID-19 persistente e lesão orgânica definitiva. Adicionalmente, outras situações podem gerar quadros similares em pacientes recuperados, como efeitos colaterais de medicamentos, complicações da doença, consequências psicológicas pós-enfermidade e infecções por outros agentes patológicos. Sendo assim, há a necessidade de estabelecer critérios diagnósticos para a SPC (RAVEENDRAN AV, 2021).

Até o momento, com relação às alterações pulmonares, estudos dos achados tomográficos apresentaram alterações semelhantes: opacidade em vidro fosco, consolidações focais e opacidades mistas, com acometendo bilateral e multifocal, distribuídos na periferia e predomínio nos campos pulmonares médios, inferiores e posteriores. Além disso, em quadros agravados e avançados, observa-se o espessamento septal e alterações reticulares sobrepostas às alterações alveolares, ou seja: acometimento intersticial pulmonar 
(ARAÚJO-FILHO JAB, et al., 2020). Mesmo após a recuperação, essas alterações radiológicas podem não sofrer resolução completa e evoluir para uma forma pulmonar residual, deixando sequelas, de gravidade variável, como exemplo a fibrose pulmonar (GEORGE PM, et al., 2020; RAI DK, et al., 2021).

Segundo as diretrizes da British Thoracic Society, os pacientes com COVID-19 devem realizar testes de função pulmonar, após 3 meses da alta hospitalar, caso ainda apresentem sintomas respiratórios contínuos ou progressivos e as alterações radiológicas de tórax não tenham se resolvido satisfatoriamente. Já a Sociedade Espanhola de Pneumologia e Cirurgia Torácica orienta a realização da espirometria simples e da medida da capacidade de Difusão Pulmonar para o Monóxido de Carbono (DLCO) na abordagem inicial dos pacientes com sintomas residuais pós-COVID-19, e inclui a pletismografia na suspeita de Doença Pulmonar Intersticial (DPI) (FONSECA A, et al., 2021).

De acordo com George PM, et al. (2020), pacientes que tiveram pneumonia grave por COVID 19, se dentro de 4 a 6 semanas após a alta hospitalar apresentarem sintomas respiratórios persistentes, novos ou progressivos, devem realizar radiografia de tórax, teste completo de função pulmonar, teste de caminhada com avaliação da saturação de oxigênio, ecocardiograma e colher amostra de escarro para análise microbiológica. Aos que apresentarem alterações radiológicas significativas ou qualquer déficit funcional nos demais exames, será necessária investigação com Tomografia Computadorizada de Alta Resolução (TCAR) e em alguns casos angiografia pulmonar por Tomografia Computadorizada (TC). Dessa forma, os pacientes com evidências de DPI, como a fibrose pulmonar, deverão ser encaminhados aos serviços regionais especializados.

Raveendran AV (2021) propôs um sistema de critérios diagnósticos para a síndrome pós-COVID-19, em que são avaliados três critérios: evidência de infecção prévia pelo SARS-CoV-2 nas 2-4 semanas anteriores, presença de sintomas que não possam ser atribuídos a outras causas e a duração do quadro. Dessa forma, a SPC pode ser diagnosticada, tendo como bases as manifestações clínicas, resultados de testes para COVID-19, como a Reação da Transcriptase Reversa seguida pela Reação em Cadeia da Polimerase (RTPCR) de swab nasofaríngeo e de anticorpos, exames de imagem (raio $\mathrm{X}$ ou tomografia computadorizada de tórax), histórico de contato com casos confirmados ou suspeitos da doença e a persistência sintomática por mais de 2 semanas em casos leves, mais de 4 semanas em casos moderados e graves e 6 semanas em casos muito graves.

Segundo Pavli A, et al. (2021), o manejo dos pacientes com a síndrome pós-COVID-19 deve ser realizado de maneira pragmática e com base nos sintomas, sem investigação excessiva. Ademais, deve-se excluir complicações sérias e causas alternativas das manifestações apresentadas pelo paciente e todo sinal ou sintoma novo precisa ser averiguado, já que eles podem ser indicativos de sequelas tardias, como problemas cardiovasculares ou pneumonia intersticial. Para determinar as causas do quadro do indivíduo acometido, o profissional tem a possibilidade de solicitar exames como hemograma completo, dosagem de eletrólitos, função renal e hepática, troponina, proteína $C$ reativa, creatinina quinase, dímero $D$, peptídeos natriuréticos cerebrais e ferritina, para avaliação de estado inflamatório e pró-trombótico, e outros testes como eletrocardiografia, raio $\mathrm{X}$ de tórax e exames de urina.

\section{Tratamento}

A síndrome pós-COVID-19 possui diversas manifestações clínicas e sua evolução varia de acordo com inúmeros fatores associados ao indivíduo. Por isso, para combatê-la ou, ao menos, minimizá-la, uma abordagem multifacetada é fundamental. Atualmente, apesar de, ainda, não haver uma padronização nas condutas, o acompanhamento, monitorização e encaminhamento dos pacientes à equipe especializada têm sido as práticas mais frequentes (IQBAL FM, et al., 2021).

Dentre as opções de tratamento em investigação, há as terapias imunomoduladoras, que atuam estimulando o sistema imunológico na ação contra o novo coronavírus ou bloqueando as vias inflamatórias. Sabe-se que, particularmente, a inibição de TGF- $\beta$, fica na interseção entre a inflamação, imunossupressão e fibrose e, sendo assim, pode servir como um eixo para facilitar o planejamento de novas estratégias direcionadas para a prevenção das sequelas provocadas pela COVID-19 (ORONSKY B, et al., 2021). 
É importante destacar que as estratégias de manejo para o tratamento da síndrome pós-COVID-19 têm o objetivo de atenuar o risco de complicações e, além disso, possuem uma grande variação, já que dependem do perfil sintomático de cada paciente. Por exemplo, alguns pacientes podem desenvolver manifestações tromboembólicas, sendo orientado o tratamento com anticoagulantes em doses terapêuticas caso seja confirmada a presença de trombose (NALBANDIAN A, et al., 2021).

Dentre as diversas manifestações neurológicas provocadas pela COVID-19, a cefaleia é uma das mais comuns. Para este tipo de complicação, será realizada uma avaliação por imagem e, em seguida, o encaminhamento ao especialista em cefaleia refratária. Já no caso dos pacientes que apresentam alterações cognitivas, uma avaliação neuropsicológica adicional se faz necessária. Por fim, para diagnosticar depressão, distúrbio do sono, ansiedade, disautonomia e/ou fadiga, instrumentos de triagem padrão podem ser utilizados (GEORGE PM, et al., 2020 apud NALBANDIAN A, et al., 2021).

Um sintoma comum em pacientes pós-COVID-19 é a tosse persistente. Mesmo não havendo comprovação científica para o quadro em específico, foi relatado o uso de mel e antitussígenos centrais (opioides); há também o uso de outros medicamentos como antimuscarínicos, anti-inflamatórios e neuromoduladores para amenizar tal sintoma (SONG WJ, et al., 2021). É importante ressaltar que as pesquisas sobre as causas e tratamentos para os sintomas longos ainda estão em andamento. Acredita-se que, para dar o suporte necessário aos pacientes em recuperação, é primordial que eles sejam atendidos em clínicas multidisciplinares, com acompanhamento sistemático, e vinculados a programas que abarcam reabilitação cognitiva, emocional e física (YELIN D, et al., 2021).

Existem inúmeros outras manifestações sistêmicas, com seus respectivos tratamentos, que poderiam ser abordados aqui. No entanto, exatamente pelo fato de no "COVID longo" haver uma miríade de possibilidades e, além disso, não existir uma fisiopatologia muito bem definida, o tratamento deve seguir uma lógica de multiespecialidade médica e profissional, como fisioterapeutas e psicólogos, por exemplo. Portanto, o melhor tratamento dependerá da atuação do profissional que estará mais hábil para atender o sinal e sintoma prevalente, em específico, que está comprometendo a qualidade de vida do paciente (VEHAR S, et al., 2021).

\section{CONSIDERAÇÕES FINAIS}

A SPC é causada pela infecção do vírus SARS-CoV-2, possui uma prevalência de até $80 \%$ em indivíduos hospitalizados e apresenta uma característica multissistêmica. As manifestações mais comuns incluem dispneia, fadiga e dor torácica e suas principais complicações englobam fibrose pulmonar, embolia pulmonar e isquemia cardioembólica aguda do membro. Em relação ao tratamento, deve ser conduzido por uma equipe multidisciplinar, com manejo dos sintomas do paciente e a realização de exames direcionados à queixa. Portanto, a SPC é um problema de saúde pública atual, com limitações referentes a dados científicos e, dessa forma, necessita de mais estudos que possam elucidar a sua abordagem.

\section{REFERÊNCIAS}

1. ANDRADE BS, et al. Long-COVID and Post-COVID health complications: an up-to-date review on clinical conditions and their possible molecular mechanisms. Viruses, 2021; 13: 700.

2. ARAÚJO-FILHO JAB, et al. Pneumonia por COVID-19: qual o papel da imagem no diagnóstico?. Jornal Brasileiro de Pneumologia, 2020; 46: e20200114.

3. BECKER RC. COVID-19 and its sequelae: a platform for optimal patient care, discovery and training. Journal of Thrombosis and Thrombolysis, 2021; 51: 587-594.

4. FAJGENBAUM D, JUNE C. Cytokine storm. New England Journal of Medicine, 2020; 383: 2255-2273.

5. FONSECA A, et al. Avaliação da função pulmonar em pacientes pós-COVID-19-quando e como devemos realizá-la? Jornal Brasileiro de Pneumologia, 2021; 47: e20210065.

6. GEORGE PM, et al. Respiratory follow-up of patients with COVID-19 pneumonia. Thorax, 2020; 75: $1009-1016$.

7. HIGGINS V, et al. COVID-19: from an acute to chronic disease? Potential long-term health consequences. Critical Reviews in Clinical Laboratory Science, 2021; 58: 297-310.

8. IQBAL FM, et al. Characteristics and predictors of acute and chronic post-COVID syndrome: A systematic review and meta-analysis. EClinicalMedicine, 2021; 36: 1-13. 
9. JOHN AE, et al. COVID-19 and pulmonary fibrosis: A potential role for lung epithelial cells and fibroblasts. Immunological Reviews, 2021; 302: 228-240.

10. KHUNTI K, et al. Long COVID - metabolic risk factors and novel therapeutic management. Nature Reviews Endocrinology, 2021; 17: 379-380.

11. KOROMPOKI E, et al. Epidemiology and organ specific sequelae of post-acute COVID-19: A narrative review. Journal of Infection, 2021; 83: 1-16.

12. MALIK P, et al. Post-acute COVID-19 syndrome (PCS) and health-related quality of life (HRQLL) - a systematic review and meta-analysis. Journal of Medical Virology, 2022; 94: 253-262.

13. MARTIMBIANCO ALC, et al. Frequency, signs and symptoms, and criteria adopted for long COVID-19: A systematic review. International Journal of Clinical Practice, 2021; 75: e14357.

14. MARTÍNEZ WC, et al. Post-COVID-19 neurological syndrome: Implications for sequelae's treatment. Journal of Clinical Neuroscience, 2021; 88: 219-225.

15. MEIER $P$, et al. Referral patterns and outcomes in noncritically ill patients with hospital-acquired acute kidney injury. Clin. J. Am. Soc. Nephrol, 2011; 6: 2215-2225.

16. MONTANI D, et al. Multidisciplinary approach for post-acute COVID-19 syndrome: time to break down the walls. European Respiratory Journal, 2021; 58: 2101090.

17. NALBANDIAN A, et al. Post-acute COVID-19 syndrome. Nature Medicine, 2021; 27: $601-615$.

18. ORGANIZAÇÃO MUNDIAL DA SAÚDE (OMS). WHO Coronavirus (COVID-19) Dashboard. 2021. Disponível em: https://covid19.who.int/. Acesso em 30 out 2021.

19. ORONSKY B, et al. A review of persistent post-COVID syndrome (PPCS). Clinical Reviews in Allergy \& Immunology, 2021; 1: 1-9.

20. PAVLI A, et al. Post-COVID syndrome: Incidence, clinical spectrum, and challenges for primary healthcare professionals. Archives of Medical Research, 2021; 52: 575-581.

21. POENARU S, et al. COVID-19 and post-infectious myalgic encephalomyelitis/chronic fatigue syndrome: a narrative review. Therapeutic Advances in Infectious Disease, 2021; 8: 20499361211009385.

22. RAI DK, et al. Post covid 19 pulmonary fibrosis - is it real threat? Indian Journal of Tuberculosis, 2021; 68: 330-333.

23. RAVEENDRAN AV. Long COVID-19: challenges in the diagnosis and proposed diagnostica criteria. Diabetes \& Metabolic Syndrome, 2021; 15: 145-146.

24. RELLO J, et al. Post-acute COVID-19 Syndrome (PACS): A public health emergency. Anaesthesia, Critical Care \& Pain Medicine, 2021; 40: 100882.

25. SATTERFIELD BA, et al. Cardiac involvement in the long-term implications of COVID-19. Nature Reviews Cardiology, $2021 ; 1: 1-10$.

26. SONG WJ, et al. Confronting COVID-19- associated cough and the post-COVID syndrome: role of viral neurotropism, neuroinflammation, and neuroimmune responses. The Lancet Respiratory Medicine, 2021; 9: 533-544.

27. SYKES DL, et al. Post-COVID-19 symptom burden: what is long-COVID and how should we manage it? Lung, 2021; 199: 113-119.

28. TOLBA M, et al. Assessment and characterization of post-COVID-19 manifestations. International Journal of Clinical Practice, 2021; 75: e13746.

29. VEHAR S, et al. Post-acute sequelae of SARS-CoV-2 infection: Caring for the 'long-haulers'. Cleveland Clinic Journal of Medicine, 2021; 88: 267-272.

30. YELIN D, et al. Long COVID-19 - it's not over until? Clinical Microbiology and Infection, 2021; 27: 506-508.

31. ZACHARIAS H, et al. Rheumatological complications of COVID-19. Autoimmunity Reviews, 2021; $20: 102883$. 